\title{
ANÁLISIS DE LA GENERACIÓN DE RESIDUOS DE APARATOS ELÉCTRICOS Y ELECTRÓNICOS (RAEES). ESTUDIO DE CASO EN LA CIUDAD DE NEIVA
}

\section{ANALYSIS OF THE GENERATION OF ELECTRICAL AND ELECTRONIC DEVICE WASTES. CASE STUDY IN THE NEIVA CITY}

\author{
Christian Felipe Valderrama Lopez ${ }^{1}$ \\ Leidy Johana Diaz ${ }^{2}$ \\ Jesus Octavio Vargas ${ }^{3}$ \\ ${ }^{1} \mathrm{Mg}$ en Higiene y Seguridad Industrial, Universidad Nacional Abierta y a Distancia \\ ${ }^{2}$ Especialista en Evaluación de proyectos, Corporación Autónoma Regional del Alto Magdalena - CAM \\ ${ }^{3}$ Especialista en Evaluación de proyectos, Fundación Universitaria Navarra - UniNavarra \\ ${ }^{1}$ christian.valderrama@unad.edu.co \\ 2 admincita@cam.gov.co \\ ${ }^{3}$ direccioningenieriaambiental@uninavarra.edu.co
}

\section{RESUMEN}

Los Residuos de Aparatos Eléctricos y Electrónicos - RAEEs se han convertido en una nueva fuente de contaminación, al punto que anualmente se están disponiendo en los rellenos sanitarios aproximadamente entre 40 y 50 millones de toneladas en países emergentes. Este tipo de residuos especiales, considerados peligrosos, son un riesgo para el medio ambiente y los seres humanos. Por lo anterior, este artículo presenta el análisis del comportamiento de la generación de residuos peligrosos en el sector industrial, comercial y de servicios en la ciudad de Neiva. Para esto se contó con el apoyo de la autoridad ambiental regional para identificar los generadores de residuos peligrosos de la ciudad y se diseñó una herramienta de recolección de información.

El procesamiento de esta información permitió identificar el tipo, la cantidad y el manejo y disposición final de este tipo de residuos en la ciudad. Se encontró que en el año 2013 se generaron $133.396 \mathrm{~kg} /$ año de RAEEs, y solo $25 \%$ de los generadores consideran a los RAEEs como peligrosos. Este estudio permite sentar las bases para dar un punto de partida a otras investigaciones asociadas con los residuos peligrosos y especiales que permita generar alternativas a mediano plazo relacionadas con la sostenibilidad, la innovación y el emprendimiento a nivel local. 
Palabras clave: Investigación descriptiva, gestión ambiental, rellenos sanitarios, residuos peligrosos, residuos de aparatos eléctricos y electrónicos.

\section{ABSTRACT}

The Waste Electrical and Electronic Equipment - WEEE has become a new source of pollution to the point that approximately 40 to 50 million tons per year are disposed in landfills in developing countries. Those types of hazardous waste are considered of special risk to the environment and humans. Hence, this research analysed the behaviour of generation of hazardous waste in industrial, commercial and service sector in the Neiva city. The regional environmental authority supported this work to identify the hazardous waste generators in the city and a tool Data collection was designed.

The processing of this information identified the waste types, quantity, handle and final disposition in the city. It found that, in 2013, WEEE was $133,396 \mathrm{~kg} / \mathrm{yr}$ and just $25 \%$ of the companies whose generated it, consider WEEE like a hazardous waste. This study lay the foundation to another researches related to hazard wastes, allowing to create new midterm opportunities related to sustainability, innovation and entrepreneurship at local level.

Keywords: Descriptive research, environmental management, landfills, hazardous waste, waste electrical and electronic equipment.

\section{CORRELATION WEEE}

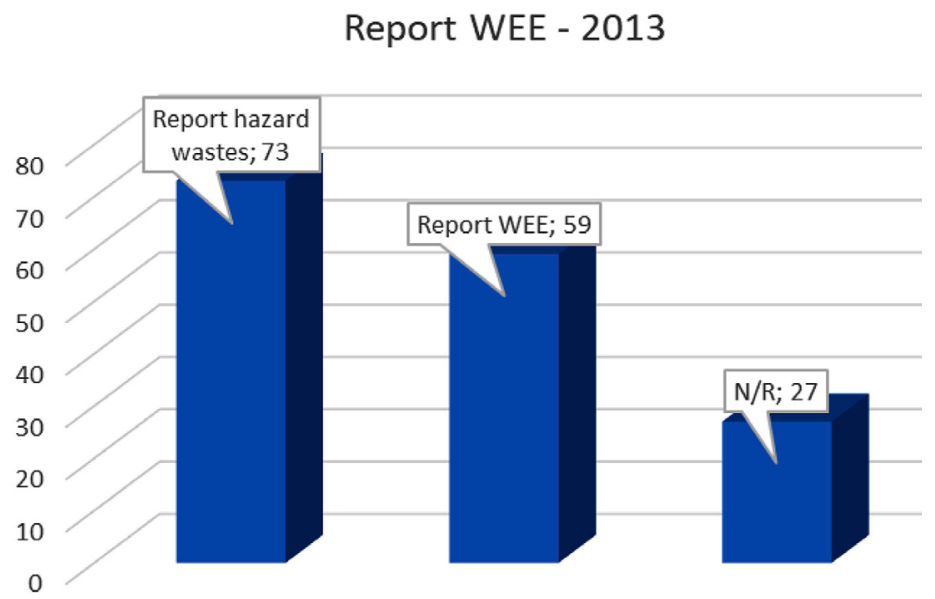

\section{CORRELATION WEEE}

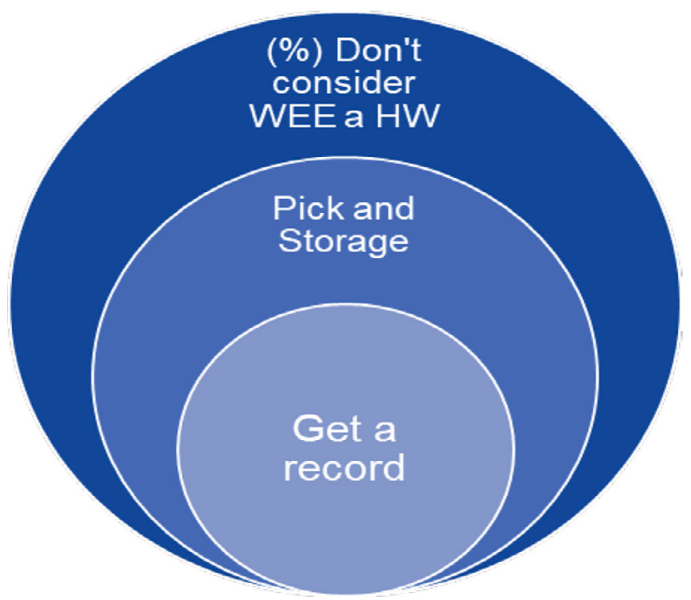

Record hazard wastes in Neiva

\begin{tabular}{l|c}
\hline Hazard Wastes & Weight report (kg) \\
\hline $\mathrm{Y}(21,22,26,29,31,41)$ & 94404,8 \\
\hline $\mathrm{A}(1020,1030,1080$, & 53,003 \\
$1160,1180)$ & $\mathbf{1 3 3 , 6 9 6}$ \\
\hline Total (kg) & \\
\hline
\end{tabular}

Graphical abstract: Waste of electrical and electronic appliances analysis. Study case in Neiva city 


\section{INTRODUCCIÓN}

El tema de los Residuos de Aparatos Eléctricos y Electrónicos (RAEEs) se ha convertido en un problema alarmante debido al rápido avance tecnológico que está afectando al mundo por la generación de las grandes cantidades de Residuos de Aparatos Eléctricos y Electrónicos (RAEEs), donde se registró un incremento anual del 5\% (Duygan \& Meylan, 2015) y al año 2012 los RAEEs generados y reportados a nivel global fueron de 48.894 millones de toneladas (Baldé, Wang, \& Huisman, 2015). El problema se agrava debido a que el consumo masivo de aparatos electrónicos como son los computadores personales y teléfonos celulares se ha incrementado y el ciclo de vida de estos se ha reducido, aumentando así la velocidad de desecho de estos equipos (Tiago, Geraldo Antônio, \& Vania Elisabete, 2017), ocupando un $8 \%$ de los residuos municipales en países como Asia e India ((Tsydenova \& Bengtsson, 2011; Widmer, Oswald-Krapf, Sinha-Khetriwal, Schnellmann, \& Böni, 2005)).

El rápido crecimiento de la población humana y el desarrollo tecnológico que someten al ambiente, está produciendo un declive cada vez más acelerado en la calidad de éste y en su capacidad para sustentar la vida dando lugar a una gran cantidad de desechos, basado principalmente en la producción de aparatos electrónicos y eléctricos mediante el uso de materiales como el plomo, estaño, cadmio, cobre, antimonio, cobalto, oro, mercurio, níquel, zinc, bario y cromo (Morf et al., 2007), los cuales al ser procesados de forma irregular generan una mayor contaminación (Manhart, 2011) y un incremento en el riesgo de la salud ocupacional y ambiental de la población por exposición a compuestos como dioxinas ((Li, Yu, Sheng, Fu, \& Peng, 2007; Tsydenova \& Bengtsson, 2011)), bifenilos policlorados (BPCs) (Wen et al., 2008), entre otros.
La realidad en Colombia no es ajena a los problemas mencionados, el Centro de Investigación de Mercados reporta que el 7\% de estos dispositivos terminan en el relleno sanitario o en manos de un reciclador (MAVDS, 2010). A pesar de existir empresas gestoras encargadas de la recolección de estos residuos, no se están llevando a cabo los lineamientos nacionales para cada una de las siguientes etapas que enmarcan la gestión integral de los Residuos de Aparatos Eléctricos y Electrónicos (RAEEs) relacionadas con el manejo, recolección, almacenamiento, etiquetado, transporte, reusó, reacondicionamiento, reutilización, reparación, reciclaje, desensamble manual y mecánico, descontaminación, fundición, refinación térmica y química, incineración y disposición final en rellenos sanitarios y rellenos de seguridad.

Por esta razón se desarrolló este estudio con el fin de investigar el comportamiento del manejo y disposición final de los RAEEs en una ciudad intermitente, como Neiva, despertando así un gran interés en los temas de investigación y emprendimiento, con el objetivo de construir una línea base que permita identificar la percepción de esta problemática y las posibles soluciones que ayuden a mitigar o prevenir los impactos que generan las practicas no adecuadas de estos residuos para el sector industrial y de esta manera, generar investigaciones en tecnologías de aprovechamiento y valorización de los mismos ajustadas a las necesidades de esta región.

\section{MATERIALES Y MÉTODOS}

En este proyecto se realizó la recolección de la información por el método de investigación descriptivo (Hernandez Sampieri, Fernandez Collado, \& Baptista Lucio, 2003). Como sistema de recolección de información se realizó el diseño, aplicación y análisis de encuestas partiendo de una matriz de análisis de 
información del Registro de Generadores de Residuos o Desechos Peligrosos del Instituto de Hidrología y Meteorología de Estudios Ambientales (IDEAM).

Se diseñó una encuesta de escala tipo frecuencia formulada en sentido positivo, constituido por 4 ítems con respuesta, dirigida al personal encargado de adelantar las acciones de manejo, recolección y disposición final con empresas gestoras de RAEEs en el municipio de Neiva. Se procedió a identificar mediante el registro de generadores de residuos o desechos peligrosos del IDEAM los usuarios inscritos de la ciudad de Neiva para el año 2013. Para establecer la muestra de estudio se utilizó un muestreo aleatorio simple con una confianza del $95 \%$ y un error máximo del $9 \%$. Se procesó la información mediante un análisis detallado para identificar el tipo y cantidad de residuos generados, además del tipo y aprovechamiento y disposición final. Para la recolección de la información se contó con el apoyo de la Corporación Autónoma Regional del Alto Magdalena - CAM.

\section{RESULTADOS Y DISCUSIÓN}

Con la información suministrada por las encuestas y las bases de datos brindadas por la Corporación Autónoma Regional del Alto Magdalena - CAM, obtenidas del aplicativo del IDEAM, se logró identificar los tipos y cantidades de residuos peligrosos de acuerdo con sus características y corriente de peligrosidad como se observa en la Tabla 1. Para la vigencia del año 2013, se encontraron 298 empresas que reportaban la generación de RAEEs llegando a un total de $133.696 \mathrm{~kg}$.

Tabla 1. Información residuos peligrosos por corriente, municipio de Neiva.

\begin{tabular}{|c|c|}
\hline Corriente de residuo o desecho peligroso & $\begin{array}{c}\text { Solido/Semisólido } \\
\text { (kg) }\end{array}$ \\
\hline Y21 - Desechos que tengan como constituyentes: compuestos de cromo hexavalente. & 875 \\
\hline Y22 - Desechos que tengan como constituyentes: compuestos de cobre. & 3.182 \\
\hline Y23 - Desechos que tengan como constituyentes: compuestos de zinc. & 592 \\
\hline Y26 - Desechos que tengan como constituyentes: cadmio, compuestos de cadmio. & 1 \\
\hline Y29 - Desechos que tengan como constituyentes: mercurio, compuestos de mercurio. & 1.599 \\
\hline Y31 - Desechos que tengan como constituyentes: plomo, compuestos de plomo. & 79.929 \\
\hline Y41 - Desechos que tengan como constituyentes: solventes orgánicos halogenados. & 7.372 \\
\hline $\begin{array}{l}\text { A1020 - Desechos que tengan como constituyentes o contaminantes, excluidos los } \\
\text { desechos de metal en forma masiva, cualquiera de las sustancias siguientes: antimonio }\end{array}$ & 1.366 \\
\hline $\begin{array}{l}\text { A1030 - Desechos que tengan como constituyentes o contaminantes cualquiera de las } \\
\text { sustancias siguientes: arsénico }\end{array}$ & 9 \\
\hline $\begin{array}{l}\text { A1080 - Residuos de desechos de zinc no incluidos en la lista B, que contengan plomo } \\
\text { y cadmio en concentraciones tales que presenten características del Anexo III. }\end{array}$ & 13 \\
\hline A1160 - Acumuladores de plomo de desecho, entero o triturado. & 24.017 \\
\hline $\begin{array}{l}\text { A1180 - Montajes eléctricos y electrónicos de desecho o restos de éstos que contengan } \\
\text { componentes como acumuladores y otras baterías incluidos en la lista A, interruptores de } \\
\text { mercurio, vidrios de tubos de rayos catódicos y otros vidrios activados y capacitadores } \\
\text { de PCB, o contaminados con constituyentes del Anexo I (por ejemplo, cadmio, mercurio, } \\
\text { plomo, bifenilo policlorado) en tal grado que posean alguna de las características del } \\
\text { Anexo III (véase la entrada correspondiente en la lista B B1110). }\end{array}$ & 14.742 \\
\hline TOTAL (kg) & 133.696 \\
\hline
\end{tabular}


Con base en la información obtenida, se tomó una muestra aleatoria simple de 95 empresas para ser encuestadas, generando los resultados arrojados a continuación.

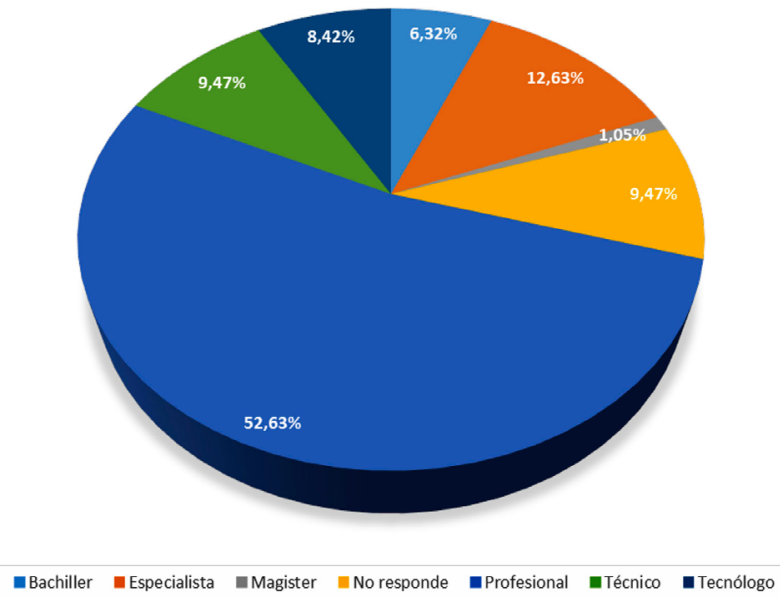

Figura 1. Nivel escolaridad del encuestado

Se encontró en la Figura 1, que el nivel de escolaridad que tienen las personas que diligenciaron las encuestas como encargados del manejo ambiental de las empresas, 52,63\% son profesionales, seguido de $12,63 \%$ que son especialistas.

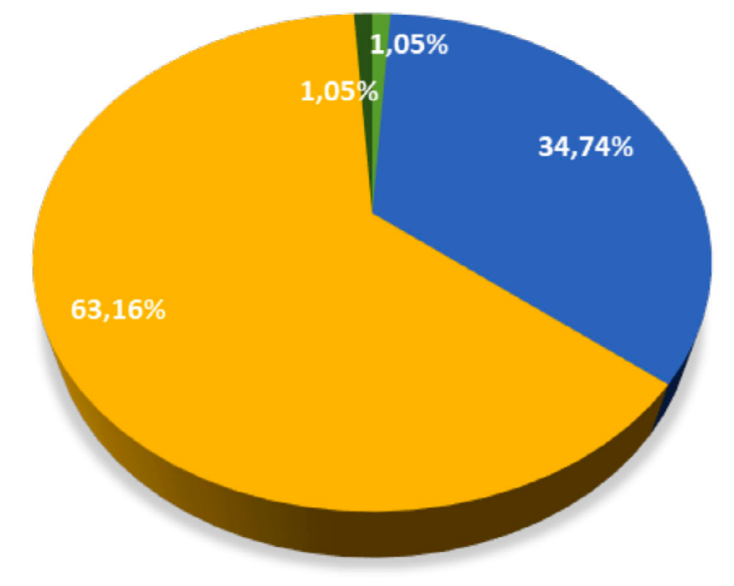

a Comercial a Industrial $\quad$ Servicios

No hay dato

Figura 2. Tipos de sectores generadores de RAEES
Como se puede observar en la Figura 2, el sector que tiene mayor representatividad es el de servicios con $63,16 \%$, seguido del industrial con $34,74 \%$ y por último, se encuentra el sector comercial.

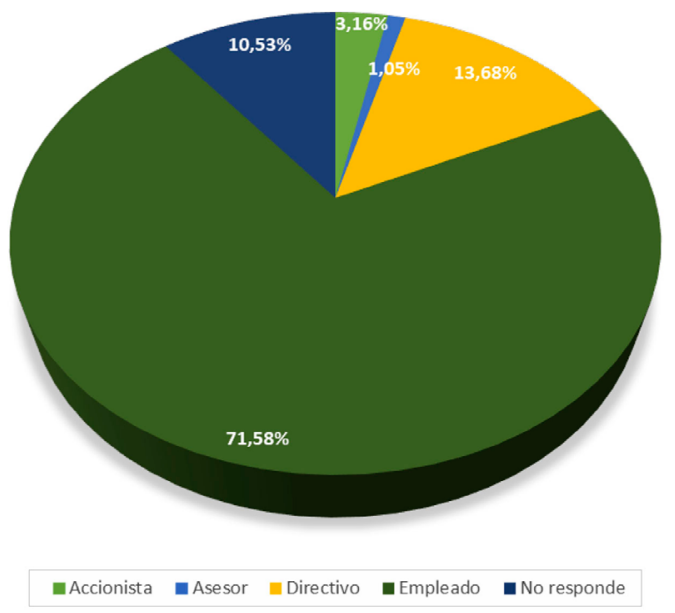

Figura 3. Nivel cargo de encuestados.

En la figura 3, se encontró que $71,58 \%$ de los encuestados corresponde a empleados, seguido de $13,68 \%$ directivos de empresas, y $10,53 \%$ no respondió que cargo ejerce dentro de la empresa.

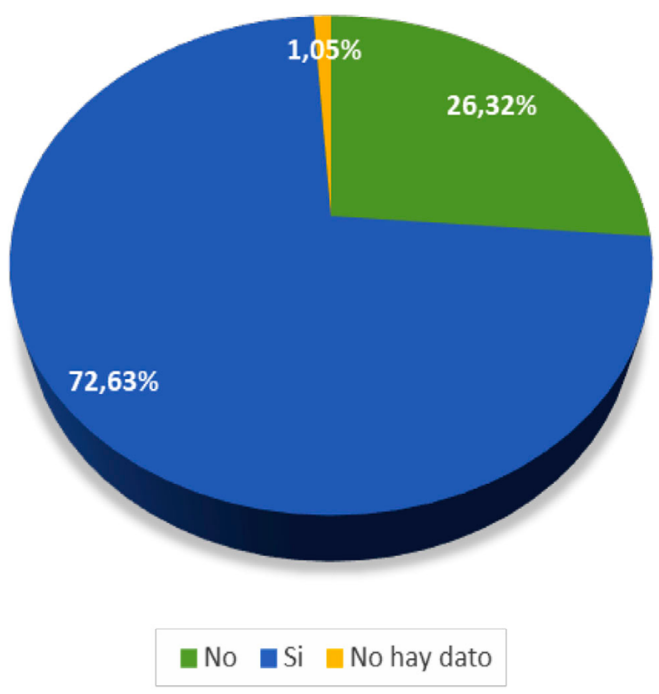

Figura 4. Planes de gestión de residuos peligrosos implementados. 
En la figura 4, se puede observar que el $72,63 \%$ de las empresas están cumpliendo con la normativa y $26,32 \%$ no está cumpliendo con la implementación de un Plan de Gestión de RES$\mathrm{PEL}$, para el porcentaje restante no hubo respuesta $(1,05 \%)$.

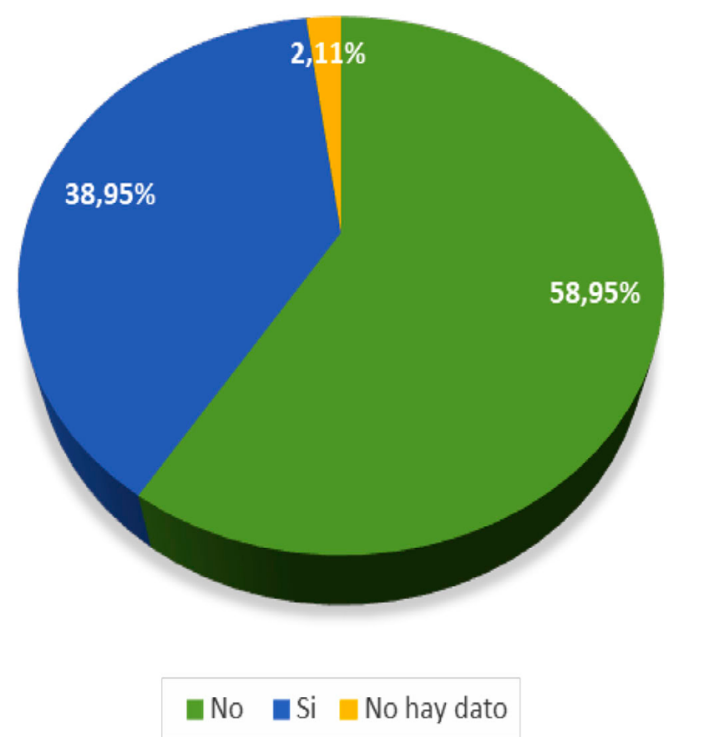

Figura 5. Recolección y almacenamiento de los RAEEs.

En la figura 5, Se evidencia que hay una población del 58,95\% que no está realizando la recolección ni el almacenamiento de los RAEEs.

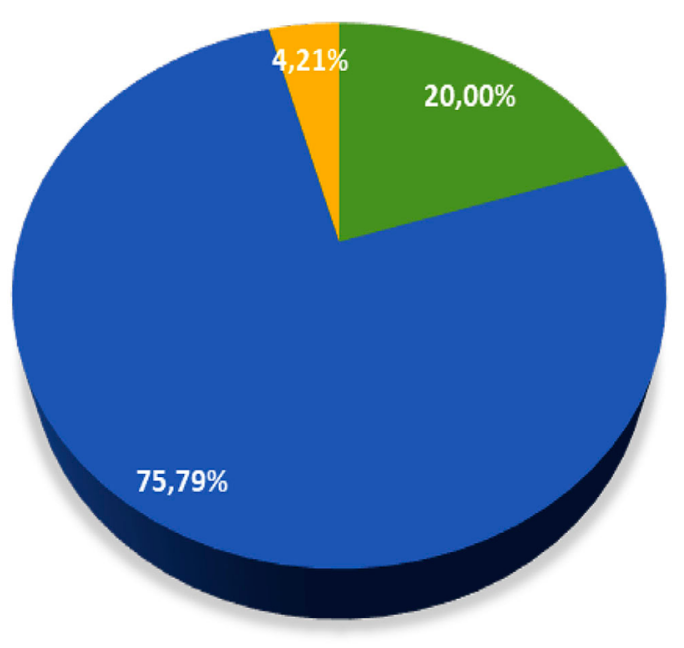

- Si No No hay dato
De acuerdo con la figura 6 , solo $20,00 \%$ de los encuestados diligencian la información, mientras que el $75,79 \%$ de la población objetivo no llevan ningún registro de estos RAEEs. Por lo anterior, es preocupante la ausencia de control sobre estos residuos como peligrosos.

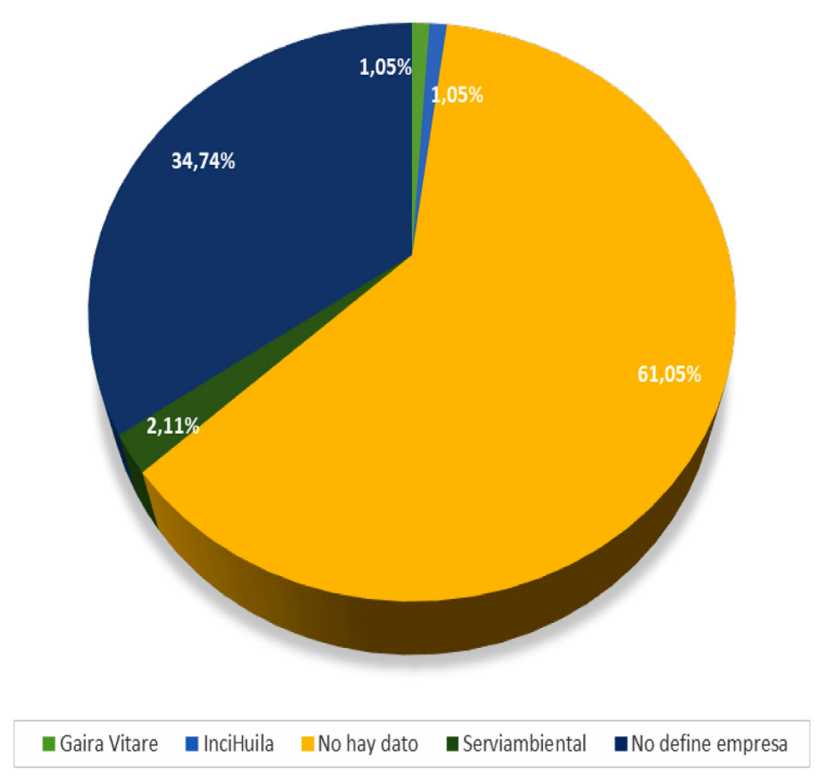

Figura 7. ¿Con qué empresa realiza la disposición final de los RAEE?

En la figura 7, se observa que en el momento de la disposición y/o la entrega de los RAEEs, solo $4,21 \%$ de los encuestados entregan estos residuos a las empresas gestoras, pero desconocen acerca de la capacidad técnica de las compañías recolectoras y la autorización por parte de la Corporación Autónoma Regional para la recolección y disposición final de estos residuos, como son SERVIAMBIENTAL e INCIHUILA.

Figura 6. ¿Diligencia el formato de generación de RAEE? 


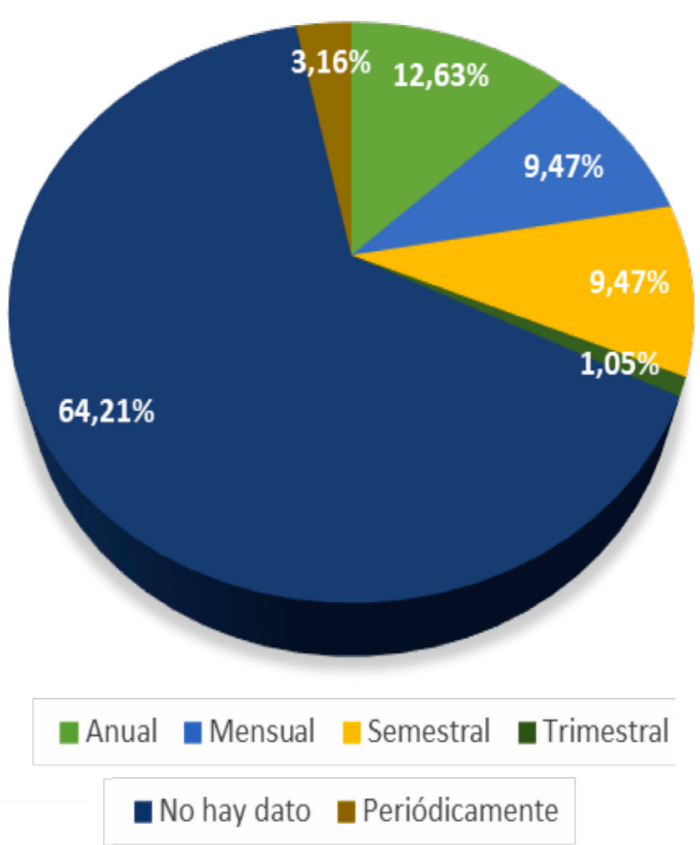

Figura 8. Periodicidad de recolección de los RAEEs.

La figura 8, muestra que $35,78 \%$ de la población objetivo realiza la recolección de los RAEEs, pero falta conciencia por parte de las empresas que consideran sin importancia la disposición final adecuada de este tipo de residuos.

\section{DISCUSIÓN}

La importancia de analizar la percepción y la clasificación de los diferentes tipos de residuos peligrosos son la clave para una sociedad moderna y sostenible, en donde se logre reducir la demanda de los productos y se fortalezcan los comportamientos de los consumos responsables (Ibanescu, Cailean Gavrilescu, Teodosiu, \& Fiore, 2018). Es por ello que esta investigación permitió identificar el nivel de información que manejan los trabajadores en el campo ambiental; sus fortalezas y debilidades que servirán como línea base para generar estrategias y futuras investigaciones articuladas con este sector en la ciudad.

La generación y reporte de $133.696 \mathrm{~kg}$ de residuos peligrosos en la ciudad es un buen indicador para poder identificar los impactos ambientales y sanitarios que pueden tener estas sustancias químicas, es por ello que se deben empezar a plantear estrategias de tratamiento y disposición asociados a los residuos electrónicos como plantas locales que generen rentabilidad económica y ambiental para la ciudad (Zeng et al., 2015), y a la vez se reduzca la obtención de recursos primarios dentro de la ciudad por su auto producción interna (Manhart, 2011).

Por otro lado, se observó una correlación entre la coherencia con el análisis de recolección y almacenamiento de los RAEEs, en donde $75 \%$ no consideran a los RAEEs como RESPEL; sin embargo, 39\% hace la recolección y almacenamiento, pero solo $23 \%$ de los que recolectan tienen un formato de generación de RAEEs en sus empresas, identificando que el flujo de estos residuos se puede estar dirigiendo a los rellenos sanitarios o a los recicladores informales que van a ocasionar un impacto más grave al medio ambiente por la liberación de residuos peligrosos sin medidas de control.

Los resultados evidenciaron la problemática ambiental en recolección, manejo y disposición final de este tipo de residuos, pues no existe ningún tipo de control por parte de ellas, ya que desconocen que este tipo de residuos contiene sustancias peligrosas. Las actividades de aprovechamiento como el reacondicionamiento y desmontaje se están realizando por empresas informales no autorizadas o por aquellas que, pese a que tienen la licencia ambiental para el almacenamiento, tratamiento y disposición final de residuos peligrosos, dicha autorización no contempla el aprovechamiento, valorización y disposición de RAEEs, perdiéndose oportunidades de generar rentabilidades y trabajar por la disminución del impacto al medio ambiente a través de una correcta disposición (Zeng et al., 2015). 
Por último, es de considerar que esta primera etapa permitió identificar la problemática actual en los diferentes actores de la ciudad; sin embargo, se debe considerar realizar un estudio a fondo que incluya a los habitantes a través de una perspectiva de pos consumo o logística inversa como se consideró en la ciudad de Sao Paulo (Angela Cassia, Wanda Maria Risso, \& Maria Eugenia Gimenez, 2015) para considerar a todos los actores y diseñar estrategias más aterrizadas a la problemática ambiental y cultural del territorio.

\section{CONCLUSIONES}

La generación de residuos de aparatos eléctricos y electrónicos son una problemática que se presenta en la ciudad de Neiva, ya que, pese a la existencia de mecanismos de recolección y control de la información por parte de la autoridad ambiental, prevalece el desconocimiento en el sector industrial que hace que su gestión no sea adecuada.

Se observó que, aunque $73 \%$ de las empresas encuestadas tienen y ejecutan un Plan de Gestión Integral de Residuos Peligrosos, $59 \%$ de estas no contempla dentro de éste el manejo ni la disposición final adecuada de Ios RAEEs. En conclusión, esta puede ser una de las causas que genera el aumento de los impactos negativos para el medio ambiente y la salud, ya que se manejan como residuos ordinarios y muchos de ellos terminan su ciclo en rellenos sanitarios, vertederos y campos a cielo abierto, convirtiéndose en residuos peligrosos.

\section{AGRADECIMIENTOS}

Agradecimientos a la Corporación Autónoma Regional del Alto Magdalena por haber facilitado el espacio y la disposición para coordinar con el sector industrial la adquisición de la información.

\section{LITERATURA CITADA}

Angela Cassia, R., Wanda Maria Risso, G., \& Maria Eugenia Gimenez, B. (2015). Estimativa da geração de resíduos de equipamentos elétricos e eletrônicos de origem domiciliar: proposição de método e aplicação ao município de São Paulo, São Paulo, Brasil / Evaluation of Waste of Electric and Electronic Equipments generation from house. Engenharia Sanitaria e Ambiental VO - 20, (3), 437. https://doi.org/10.1590/S141341522015020000133701

Baldé, C. P., Wang, F., \& Huisman. (2015). The Global E-waste Monitor 2014. (United Nations University, Ed.). Bonn. Retrieved from http://i.unu. edu/media/ias.unu.edu-en/news/7916/Global-Ewaste-Monitor-2014-small.pdf

Duygan, M., \& Meylan, G. (2015). Strategic management of WEEE in Switzerland-combining material flow analysis with structural analysis. Resources, Conservation and Recycling, 103, 98109. https://doi.org/https://doi.org/10.1016/j. resconrec.2015.06.005

Hernandez Sampieri, R., Fernandez Collado, C., \& Baptista Lucio, P. (2003). Metodologia de la investigación. (Mc GrawHill, Ed.). Mexico.

Ibanescu, D., Cailean Gavrilescu, D., Teodosiu, C., \& Fiore, S. (2018). Assessment of the waste electrical and electronic equipment management systems profile and sustainability in developed and developing European Union countries. Waste Management (New York, N.Y.), 73, 39-53. https://doi.org/10.1016/j.wasman.2017.12.022

IDEAM. (2013). Portal generadores RAEES. Retrieved from http://kuna.ideam.gov.co/mursmpr/ index.php

Li, H., Yu, L., Sheng, G., Fu, J., \& Peng, P. (2007). Severe PCDD/F and PBDD/F Pollution in Air around an Electronic Waste Dismantling Area in China. Environmental Science \& Technology, 41(16), 5641-5646. https://doi.org/10.1021/ es0702925.

Manhart, A. (2011). International Cooperation for Metal Recycling From Waste Electrical and Electronic Equipment: An Assessment of the "Bestof-Two-Worlds" Approach. Journal of Industrial Ecology, 15(1), 13-30. https://doi.org/10.1111/ j.1530-9290.2010.00307.x 
MAVDS. (2010). Lineamientos Técnicos para el Manejo de Residuos de Aparatos Eléctricos y Electrónicos. Bogota. Retrieved from http:// www.residuoselectronicos.net/wp-content/ uploads/2012/03/Guia_RAEE_MADS_2011-reducida.pdf

Morf, L. S., Tremp, J., Gloor, R., Schuppisser, F., Stengele, M., \& Taverna, R. (2007). Metals, non-metals and PCB in electrical and electronic waste - Actual levels in Switzerland. Waste Management, 27(10), 1306-1316. https://doi. org/10.1016/j.wasman.2006.06.014

Tiago, P., Geraldo Antônio, R., \& Vania Elisabete, S. (2017). Avaliação da geração de resíduos de equipamentos eletroeletrônicos (REEEs) em uma universidade particular / Evaluation of the electrical and electronic equipment waste (WEEE) generation in a private university. Engenharia Sanitaria e Ambiental VO - 22, (4), 625. https:// doi.org/10.1590/s1413-41522017142636

Tsydenova, O., \& Bengtsson, M. (2011). Chemical hazards associated with treatment of waste electrical and electronic equipment. Waste Management, 31(1), 45-58. https://doi.org/10.1016/J. WASMAN.2010.08.014
Wen, S., Yang, F.-X., Gong, Y., Zhang, X.-L., Hui, Y., Li, J.-G., ... Xu, Y. (2008). Elevated levels of urinary 8-hydroxy-2'-deoxyguanosine in male electrical and electronic equipment dismantling workers exposed to high concentrations of polychlorinated dibenzo-p-dioxins and dibenzofurans, polybrominated diphenyl ethers, and polychlorinated biphenyls. Environmental Science \& Technology, 42(11), 4202-7. Retrieved from http://www.ncbi.nlm.nih.gov/pubmed/18589988

Widmer, R., Oswald-Krapf, H., Sinha-Khetriwal, D., Schnellmann, M., \& Böni, H. (2005). Global perspectives on e-waste. Environmental Impact Assessment Review, 25(5), 436-458. https://doi. org/10.1016/J.EIAR.2005.04.001

Zeng, X., Song, Q., Li, J., Yuan, W., Duan, H., \& Liu, L. (2015). Solving e-waste problem using an integrated mobile recycling plant. Journal of Cleaner Production, 90, 55-59. https://doi.org/10.1016/J. JCLEPRO.2014.10.026
Conflicto de Intereses Los autores declaran no tener ningún conflicto de intereses 
